\title{
Smooth quantum-classical transition in photon subtraction and addition processes
}

\author{
A. V. Dodonov and S. S. Mizrahi \\ Departamento de Fúsica, Universidade Federal de São Carlos, \\ P.O. Box 676, São Carlos, 13565-905, São Paulo, Brazil
}

\begin{abstract}
Recently Parigi et al. [Science 317, 1890 (2007)] implemented experimentally the photon subtraction and addition processes from/to a light field in a conditional way, when the required operations were produced successfully only upon the positive outcome of a separate measurement. It was verified that for a low intensity beam (quantum regime) the bosonic annihilation operator $a$ does indeed describe a single photon subtraction, while the creation operator $a^{\dagger}$ describes a photon addition. Nonetheless, the exact formal expressions for these operations do not always reduce to these simple identifications, and in this connection here we deduce the general superoperators for multiple photons subtraction and addition processes and analyze the statistics of the resulting states for classical field states having an arbitrary intensity. We obtain closed analytical expressions and verify that for classical fields with high intensity (classical regime) the operators that describe photon subtraction and addition processes deviate significantly from simply $a$ and $a^{\dagger}$. Complementarily, we analyze in details such a smooth quantum-classical transition as function of beam intensity for both processes.
\end{abstract}

PACS numbers: 03.65.Ta; 42.50.Ar; 42.50.Lc

\section{INTRODUCTION}

It is well known [1] that the probability for absorbing one photon per unit time from an electromagnetic field is proportional to the average value of the ordered product of the negative and positive frequency electric field operators over some quantum state $\rho$, also known as the statistical operator. In the simplest case of a single-mode, that probability can be expressed in terms of the standard bosonic 'annihilation' and 'creation' operators $a$ and $a^{\dagger}$, satisfying the commutation relation $\left[a, a^{\dagger}\right]=1$, as

$$
P=\gamma \operatorname{Tr}\left(a \rho a^{\dagger}\right),
$$

where $\rho$ stands for the field state just before absorption and $\gamma$ is an appropriate coefficient. After interacting with some detector that absorbs one photon, the field makes a transition to a new state, which can be formally described by the action of a photon subtraction superoperator (PSS) $\mathcal{D}$ as [2]

$$
\rho^{\prime}=P^{-1} \mathcal{D} \rho,
$$

where $\rho^{\prime}$ represents the field state immediately after the subtraction of one photon and $P=\operatorname{Tr}(\mathcal{D} \rho)$ is the probability for that process.

The hermiticity of $\rho^{\prime}$ is always assured whenever $\mathcal{D}$ takes the form

$$
\mathcal{D} \rho \equiv \gamma O \rho O^{\dagger}
$$

where $O$ is some operator responsible for the subtraction of one photon from the field and the explicit form of $O$ depends on the details of the field-detector interaction. Since the 1960s several models were proposed [3, 4, 5, 6] (see 7] for more references therein) and the first one 2] used the rather simple identification $O=a$, when Eq. (3) becomes

$$
\mathcal{A} \rho=\gamma a \rho a^{\dagger}
$$

and we shall refer to it as $A$-model. Although such a form, Eq. (4), seems quite natural in view of equation (1), this choice was, as a matter of fact, intuitive, although later, under certain assumptions - such as the weak coupling, low intensity and short interaction time - the authors of Refs. [5, 8] were able to derive it from doing a microscopic analysis.

Nonetheless, if those assumptions are replaced by others, one can obtain different superoperators $\mathcal{D}$. A family of PSS based on the nonlinear lowering operators of the form $O=(1+\hat{n})^{-\beta} a$, where $\hat{n}=a^{\dagger} a$, was derived in Ref. [8]. The special case $\beta=1 / 2$, called $E$-model,

$$
\mathcal{E} \rho=E_{-} \rho E_{+}, \quad E_{-} \equiv(1+\hat{n})^{-1 / 2} a
$$

was originally proposed ad hoc in [9, 10]. Later, analyzing carefully a microscopic model, it was shown [8, 11, 12] that both superoperators $\mathcal{A}$ and $\mathcal{E}$ are particular cases of a more general one, where $\mathcal{A}(\mathcal{E})$ is specific for a small (large) mean photon number.

Recently, the A-model was subdued to an experimental verification in the low photon number regime and weak field-detector coupling [13]. That experiment employed a beam-splitter and a single photon on/off detector (SPD), within a simple scheme as illustrated in the Fig. 1h: a low intensity beam, with a small mean photon number per unit time, is prepared to hit a low reflectivity beamsplitter. The reflected beam is continuously measured by the SPD, and whenever it clicks, the transmitted field statistical operator can be described, approximately, by the A-model [13].

However, as already observed in [11], $\mathcal{A}$ is an unbounded superoperator and some physical inconsistencies appear [14]. For example, $\mathcal{A}$ does not hold for large values of the initial mean photon number, since the probability of a photon subtraction, $P=\gamma \operatorname{Tr}\left(a \rho a^{\dagger}\right)$, may become larger than 1. Moreover, applying $\mathcal{A}$ on some specific field states, the calculations predict results which look counterintuitive. For example, operating with $\mathcal{A}$ on the 


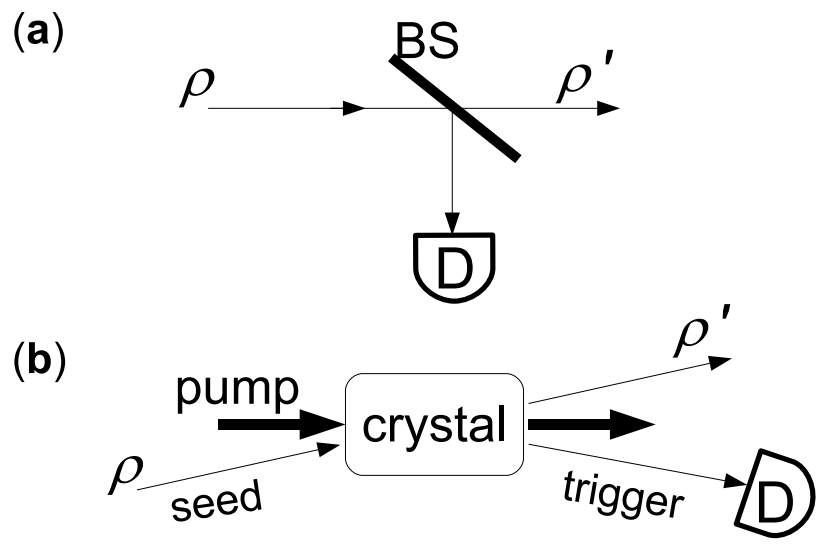

FIG. 1: (a) Scheme for conditional photon subtraction by means of a beam-splitter (BS) and a photodetector (D). (b) Scheme for conditional photon addition using a pump beam, a non-linear optical crystal and a photodetector.

thermal state, which has the photon number distribution

$$
p_{n}^{(t h)}=\frac{n_{0}^{n}}{\left(n_{0}+1\right)^{n+1}},
$$

where $n_{0}$ is the mean photon number, for post-selected state we obtain $\langle n\rangle_{\mathcal{A}}=2 n_{0}$. Although this prediction was confirmed experimentally in the 'quantum limit' of small mean photon number, $n_{0} \lesssim 1$, it is counterintuitive from the classical point of view, namely, a high intensity field cannot double the intensity due to a one-photon detection. So $\mathcal{A}$ can describe satisfactorily the low intensity field but not a high intensity one. Quite differently, using the E-model we get $\langle n\rangle_{\mathcal{E}}=n_{0}$, which is sound for a high intensity field after one-photon detection, whereas it does not reproduce the observed doubling for low intensity. Therefore, it is interesting to analyze the behavior of the PSS in the 'classical limit' (large mean photon number) and how the pattern of post-selected state statistics changes smoothly and continuously as function of the field intensity. The quantum-classical transition in photon subtraction process regards the passage between the two extreme situations for classical field states that can be prepared with arbitrary low and high intensities.

Besides the photon subtraction, one may also implement the photon addition operation by the conditional stimulated down-conversion in a non-linear optical crystal, as reported in [13] and illustrated in the Fig. 10. Inside the crystal the pumped photons may decay spontaneously into two entangled photons with energies that sum up to that of the parent one. Upon detecting one of these photons (known as the trigger photon) along a particular direction, the other photon state is unambiguously determined. If one injects a seed light into the crystal, the stimulated emission may occur into the same mode, and the detection of a single trigger photon (by means of a SPD) indicates the conditional generation of the photon-added state. Using this scheme, the one photon addition superoperator (PAS) for low beam intensity is

$$
\mathbb{A} \rho=\gamma^{\prime} a^{\dagger} \rho a
$$

(we call it the $A_{+}-$model), which was experimentally implemented for a low mean photon number [13]. Since the superoperator $\mathbb{A}$ is unbounded, with the probability of photon addition becoming larger than 1 for large field intensities, in analogy to the E-model we also define the $E_{+}$-model as

$$
\mathbb{E} \rho=E_{+} \rho E_{-},
$$

that will also be analyzed and discussed below.

In the present paper we generalize the theoretical analysis presented in [13] for both, the photon subtraction and photon addition operations, by substituting the SPD by the $k$-photon resolving [19] or nonresolving [20] detector, and obtain formally PSS and PAS valid for an arbitrary field at any intensity. Although the resulting formal expressions for the PSS and PAS are compact, in general, it is not straightforward to obtain closed analytical expressions for these operations for an arbitrary initial field state. Therefore, it is desirable to have some simple approximate expressions for the PSS and PAS that hold in a specific regime of parameters and can be easily evaluated for an arbitrary field state, providing a simple means of predicting the outcome of the experiment. In this connection, we show that in the quantum limit (small photon number), the A-model and $\mathrm{A}_{+}$-model are good approximations to the exact PSS and PAS for any field state, respectively, while in the classical limit (large photon number), the $\mathrm{E}$ - and $\mathrm{E}_{+}-$models are more appropriate for a nonresolving detector and the 'classical' field states: coherent, thermal and 'mixed light'.

The paper contains three additional sections. In section [1] we obtain exact analytical expressions for the PSS and analyze the behavior of the photon subtraction probabilities and post-selected states statistics for any intensity of the field and for different kinds of 'classical' field states: (a) coherent, (b) thermal and (c) 'mixed light' 21], which, in principle, can be produced in the laboratory with arbitrarily low or high beam intensities. In section [III we do the same for the PAS, considering (a) coherent and (b) thermal field states. In section [V] we present a summary and our conclusions.

\section{PHOTON SUBTRACTION}

After passing through the beam-splitter a small fraction of the incident field (signal) is reflected into the mode $b$, initially in the vacuum state $\left|0_{b}\right\rangle\left\langle 0_{b}\right|$ (Fig. 1 1 ). The post-selection procedure - in which $k>0$ photons are detected by a detector placed in the reflected path - allows to express the transmitted field state as Eq. (2), where the exact PSS is written as

$$
\mathcal{D}_{k} \rho=\operatorname{Tr}_{b}\left[M_{k} U\left(\rho \otimes\left|0_{b}\right\rangle\left\langle 0_{b}\right|\right) U^{\dagger}\right],
$$


where $\rho$ is the incident field state. Here

$$
U=\exp \left[\theta\left(a^{\dagger} b-a b^{\dagger}\right)\right]
$$

is the beam-splitter operator, where $a$ (signal beam) and $b$ (reflected beam) represent the two modes resulting from the incident beam, and $\theta$ is a parameter related to the reflectivity $\left(R=\sin ^{2} \theta\right)$ and transmittivity $\left(T=\cos ^{2} \theta\right)$ coefficients. The operator $M_{k}=\sum_{l=k}^{\infty} \Upsilon_{l}\left|l_{b}\right\rangle\left\langle l_{b}\right|$, that acts on the reflected mode, stands for the action of the detector (referred as $\mathcal{D} k$, for short). $\Upsilon_{l}=1$ for a nonresolving $k$-photon detector $(N \mathcal{D} k)$, that clicks whenever $k$ or more photons are absorbed [19, 20], and $\Upsilon_{l}=\delta_{l, k}$ for the resolving $k$-photon detector $(R \mathcal{D} k)$, that clicks when exactly $k$ photons are absorbed [19]. The SPD corresponds to $N \mathcal{D} 1$ according to this notation.

The resulting PSS is a generalization of the Eq. (3)

$$
\mathcal{D}_{k} \rho=\sum_{l=k}^{\infty} \Upsilon_{l}\left\langle l_{b}|U| 0_{b}\right\rangle \rho\left\langle l_{b}|U| 0_{b}\right\rangle^{\dagger}
$$

The operator $U$ has the form $\exp \left[-\theta\left(K_{+}+K_{-}\right)\right]$where $K_{+} \equiv b^{\dagger} a, K_{-} \equiv-b a^{\dagger}$; a third operator, $K_{0} \equiv\left(b^{\dagger} b-\right.$ $\left.a^{\dagger} a\right) / 2$, is necessary to close the $\mathrm{su}(1,1)$ algebra, they constitute the generators of the $\mathrm{SU}(1,1)$ group. Using the factorization theorem [15, 16, 17, 18] we can write (11) as

$$
\mathcal{D}_{k} \rho=\sum_{l=k}^{\infty} \Upsilon_{l} \frac{\left(\tan ^{2} \theta\right)^{l}}{l !} a^{l}(\cos \theta)^{\hat{n}} \rho(\cos \theta)^{\hat{n}} a^{\dagger l} .
$$

which is the exact and complete PSS compatible with the experimental setup reported in [13]. For a small mean photon number $n_{0}$ and $R \ll 1$ one has roughly $\operatorname{Tr}\left[(\cos \theta)^{\hat{n}} \rho(\cos \theta)^{\hat{n}}\right] \sim\left(1-n_{0} R\right)$, so for $n_{0} R \ll 1$ one obtains

$$
\mathcal{D}_{k} \rho \simeq \mathcal{A}_{k} \rho \equiv \frac{R^{k}}{k !} a^{k} \rho a^{\dagger k}
$$

for both kinds of detectors. For $k=1$ one retrieves the A-model, whereas for $k>1$ we have a straightforward generalization of A-model for multiphoton subtraction.

The photon number distribution of the post-selected state for the $k$ photons detector is

$$
p_{n, \mathcal{D} k}^{\prime}=P_{\mathcal{D} k}^{-1} \Theta_{n}^{(\mathcal{D} k)},
$$

where

$$
\Theta_{n}^{(\mathcal{D} k)}=\sum_{l=k}^{\infty} \Upsilon_{l}\left(\begin{array}{c}
n+l \\
n
\end{array}\right) T^{n} R^{l} p_{n+l}
$$

and $P_{\mathcal{D} k}=\sum_{n=0}^{\infty} \Theta_{n}^{(\mathcal{D} k)}$ is the probability for $k$-photon subtraction, with $p_{n}$ denoting the initial photon number distribution.

Below we specialize to three different states of the field, which are currently produced in the lab: the coherent,

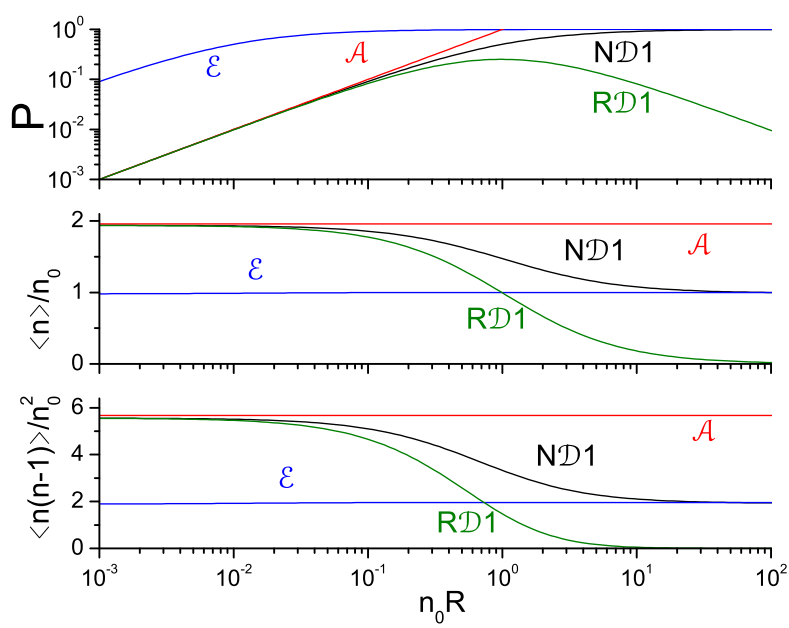

FIG. 2: (Color online) $N \mathcal{D} 1$ and $R \mathcal{D} 1$ results for the 'mixed light' state with mean photon number $n_{0}$ compared to the predictions given by superoperators $\mathcal{A}$ and $\mathcal{E}$. Here $n_{c}=n_{t} / 4$.

thermal and the 'mixed light' [21]. The latter is a 'mixture of the thermal and coherent radiation' 22], whose the photon number distribution is

$$
p_{n}^{(m l)}=\exp \left(-\frac{n_{c}}{1+n_{t}}\right) \frac{n_{t}^{n}}{\left(n_{t}+1\right)^{n+1}} L_{n}\left[-\frac{n_{c}}{n_{t}\left(1+n_{t}\right)}\right] .
$$

where $n_{c}$ and $n_{t}$ are the mean photon numbers of the coherent and thermal part of the mixed light, respectively, $n_{0}=n_{c}+n_{t}$ is the total photon number and $L_{n}(\cdot)$ is a Laguerre polynomial. For these three states the resulting expressions for $p_{n, \mathcal{D} k}^{\prime}, P_{\mathcal{D} k}$ and the two lower moments of the photon number distribution for the post-selected state are given in the appendix $\mathrm{A}$.

For a small mean photon number, $n_{0} R \ll 1$, the expressions for the photon subtraction probability and the two lower moments of photon number in the resulting state [see Eqs. in appendix A] are approximately equal to the expressions corresponding to the generalized Amodel. Thus, in the quantum regime (low intensity field), $\mathcal{A}_{k}$ is a good approximation for the PSS, and the detection of one or more photons may significantly increase the mean photon number of the post-selected state. On the other hand, for a high intensity field, $n_{0} R \gg 1$, the results are quite different from those predicted by using $\mathcal{A}_{k}$. This behavior can be appreciated looking Figs. 2 and 3 , where we have plot $P_{\mathcal{D} 1},\langle n\rangle_{\mathcal{D} 1}$ and $\langle n(n-1)\rangle_{\mathcal{D} 1}$, together with the corresponding predictions of $\mathcal{A}_{1}$ and $\mathcal{E}_{1}$, for the mixed light state with $n_{c}=n_{t} / 4$ (Fig. 2) and $n_{c}=10 n_{t}$ (Fig. 3), setting $R=10^{-2}$. One can perceive, from the figures, that for $n_{0} R \gg 1$ the probabilities and the factorial moments for $N \mathcal{D} 1$ are close to those predicted by the generalized E-model. Therefore, for the considered classical field states, in the classical regime $\mathcal{E}_{k}$ 


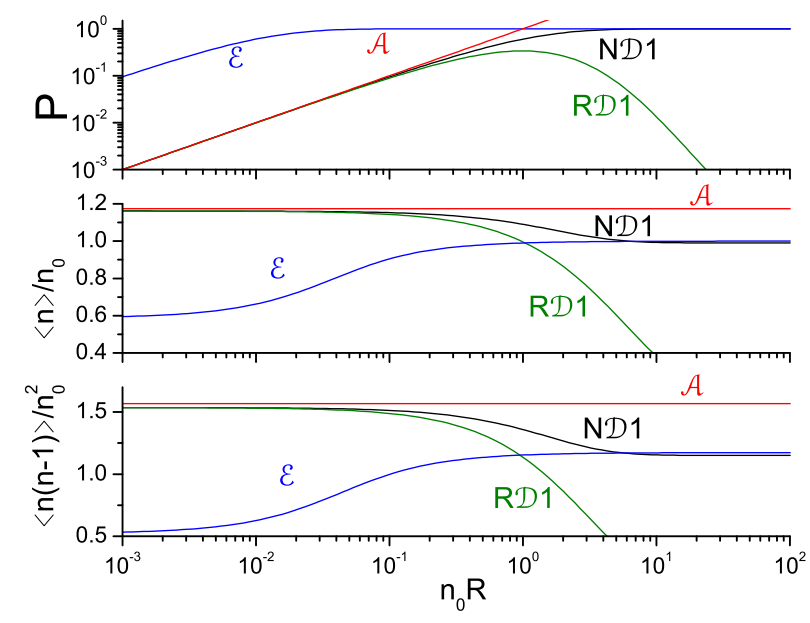

FIG. 3: (Color online) Same as Fig. 2 for $n_{c}=10 n_{t}$.

represents better the photon subtraction process (using a nonresolving detector) and, as verified empirically, the mean photon number does not increase upon the photodetection. The smooth transition between the A- and E- models occurs in the region $n_{0} R \sim 1$.

The important effect of the measurement back-action may be clearly verified by comparing the outcomes of the instantaneous $k$-photon detection (using either $N \mathcal{D} k$ or $R D k$ ) to the sequential detection of $k$ photons (one by one, as a sequence of discernible clicks), using an array of $k$ SPD's and detecting one click in each one. For the sequential $k$ photons detection the PSS is $\mathcal{S}_{k}=$ $\left(\mathcal{D}_{N 1}\right)^{k}$, where $\mathcal{D}_{N 1}$ is given by Eq. (12) with $\Upsilon_{l}=1$. The resulting expressions are given in the appendix $\mathrm{B}$ and plotted in the Fig. 4. Looking at it we can compare the outcomes of the three kinds of detectors, $\mathcal{S} 2, N \mathcal{D} 2$ and $R \mathcal{D} 2$, for the thermal state and $R=10^{-2}$. It turns out that for a sequential counting, the probability of detecting $k$ photons is always higher than that calculated by admitting an instantaneous detection. Moreover, for $n_{0} R \ll 1$ the mean photon number in the post-selected state is always higher than for instantaneous detection, while for $n_{0} R \gtrsim 1$ different mean values are predicted, such that $\langle n\rangle_{N \mathcal{D} k}>\langle n\rangle_{\mathcal{S} k}>\langle n\rangle_{R \mathcal{D} k}$, see Fig. [4

\section{PHOTON ADDITION}

The $k$-photon addition superoperator is defined as [13]

$$
\mathbb{D}_{k} \rho=\operatorname{Tr}_{b}\left[M_{k} u \rho\left|0_{b}\right\rangle\left\langle 0_{b}\right| u^{\dagger}\right],
$$

where $M_{k}=\sum_{l=k}^{\infty} \Upsilon_{l}\left|l_{b}\right\rangle\left\langle l_{b}\right|$ (as defined previously) and

$$
u=\exp \left[\lambda\left(a b-a^{\dagger} b^{\dagger}\right)\right]
$$
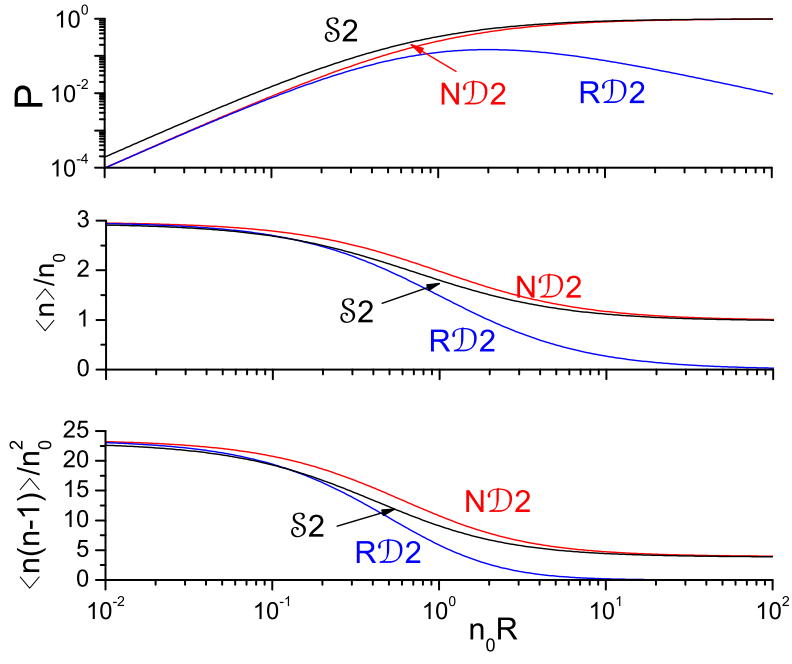

FIG. 4: (Color online) Comparison of 2 sequential detections with SPD's to the 2-photon detection using either the resolving or the nonresolving detectors, for the thermal state.

is the operator describing the parametric downconversion process with gain factor $\lambda \ll 1$. The operator $u$ contains two generators of the $s u(1,1)$ algebra, $\tilde{K}_{+}=a^{\dagger} b^{\dagger}, \tilde{K}_{-}=a b$, while the third one is $\tilde{K}_{0}=\left(a^{\dagger} a+b^{\dagger} b+1\right) / 2$. So Eq. (17) becomes [18]

$\mathbb{D}_{k} \rho=t \sum_{l=k}^{\infty} \Upsilon_{l} \frac{\left(\tanh ^{2} \lambda\right)^{l}}{l !} a^{\dagger l}\left(\cosh ^{-1} \lambda\right)^{\hat{n}} \rho\left(\cosh ^{-1} \lambda\right)^{\hat{n}} a^{l}$,

and we define $r \equiv \sinh ^{2} \lambda$ and $t \equiv \cosh ^{-2} \lambda$. For a small mean photon number one obtains

$$
\mathbb{D}_{k} \rho \simeq \mathbb{A}_{k} \rho=\frac{r^{k}}{k !} a^{\dagger k} \rho a^{k},
$$

which is the generalization of the $\mathrm{A}_{+}$-model. As like as $\mathcal{A}_{k}$, the superoperator $\mathbb{A}_{k}$ is unbounded, with the probability of photon addition becoming larger than 1 for $n_{0} \gg 1$. Therefore, the $\mathrm{A}_{+}$-model cannot stand for high field intensities. Here we shall study the behavior of the lowest photon number moments in the transition from the quantum regime, $n_{0} \ll 1$, to the classical one, $n_{0} \gg 1$, and compare the results with the predictions of the generalized $\mathrm{E}_{+}$-model, $\mathbb{E}_{k} \rho=E_{+}^{k} \rho E_{-}^{k}$. Contrarily to $\mathbb{A}_{k}$, superoperator $\mathbb{E}_{k}$ is bounded, presenting probability $P_{\mathbb{E} k}=1$ and the mean value $\langle n\rangle_{\mathbb{E} k}=n_{0}+k$ for $k$-photon addition operation.

The photon number distribution of the $k$-photon added state is given by

$$
p_{n, \mathbb{D} k}^{\prime}=P_{\mathbb{D} k}^{-1} \Theta_{n}^{(\mathbb{D} k)},
$$




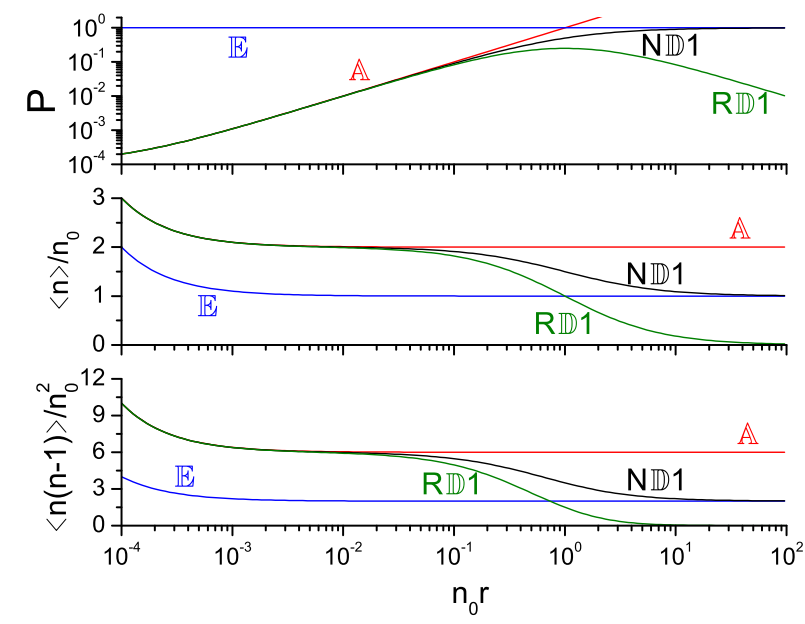

FIG. 5: (Color online) $N \mathbb{D} 1$ and $R \mathbb{D} 1$ results for the thermal state with mean photon number $n_{0}$ compared to the predictions of superoperators $\mathbb{A}$ and $\mathbb{E}$.

where

$$
\Theta_{n}^{(\mathbb{D} k)}=\sum_{l=k}^{\infty} \Upsilon_{l}\left(\begin{array}{l}
n \\
l
\end{array}\right) t^{n+1} r^{l} p_{n-l}
$$

and the probability of the $k$-photon addition is $P_{\mathbb{D} k}=$ $\sum_{n=0}^{\infty} \Theta_{n}^{(\mathbb{D} k)}$, independently of the number of photons present in the field. In order to make the physics more transparent, we assume the simplest situation $k=1$, for which $P_{\mathbb{A} 1}=r\left(n_{0}+1\right)$ and $\langle n\rangle_{\mathbb{E} 1} / n_{0}=1+n_{0}^{-1}$.

For the two different field states, the coherent and the thermal, we get the expressions given in the appendix $\mathrm{C}$ For $n_{0} r \ll 1$, in both cases, the expressions become very close to those predicted by the $\mathrm{A}_{+}$-model, so in the quantum regime the photon creation operator $a^{\dagger}$ describes accurately the photon addition process. In the regime $n_{0} r \gg 1$, for the coherent state the predictions of the $\mathrm{A}_{+-}$and $\mathrm{E}_{+-}$models for the moments $\langle n\rangle$ and $\langle n(n-1)\rangle$ are very similar, while for the thermal state, according to the $\mathrm{A}_{+}$-model, the mean photon number is roughly twice the one predicted by the $\mathrm{E}_{+}$-model. In Fig. [5] we show the behaviors of the photon addition probability $P,\langle n\rangle$ and $\langle n(n-1)\rangle$ for the thermal state as function of $n_{0} r$ for $\lambda=10^{-2}$. We see that by using a nonresolving detector for $n_{0} r \gg 1$, the expressions approach the results of the $\mathrm{E}_{+}$-model, so in the classical regime the $\mathrm{A}_{+}$-model ceases to represent the PAS and the $\mathrm{E}_{+}$-model becomes more appropriate. The transition between the $\mathrm{A}_{+-}$and $\mathrm{E}_{+}$- models occurs in the region $n_{0} r \sim 1$. Finally, notice that, although the photon resolving and nonresolving detectors are quite similar in the quantum regime, they are completely different in the classical one, as clearly seen from Figs. 2, 3, 4 and 5.

\section{SUMMARY AND CONCLUSIONS}

In summary, we have analyzed the quantum-classical transitions for the photon subtraction and for the photon addition processes from or into the field. We used the usual formal representations for the beam-splitter, the optical nonlinear crystal and the $k$-photon resolving or nonresolving detector, as it was described in 13]. We considered three classical field states - coherent, thermal and the 'mixed light' - to illustrate the transition from quantum to classical regimes and studied the photon number factorial moments in the post-selected state as function of the intensity of the prepared field, that goes on either a beam-splitter or a nonlinear crystal. We obtained the formal expressions for the PSS and the PAS, valid for any input state, and for the considered classical states we derived closed analytical expressions for the photon number distribution of the post-selected state and the associated lower factorial moments, as well as the photon addition and subtraction probabilities.

We found that in the quantum regime (small photon number) the PSS can be described approximately by the generalized A-model, while the PAS by the generalized $\mathrm{A}_{+}$-model for any field state. The mean photon number in the post-selected state may increase significantly due to the photon subtraction. On the other hand, in the classical regime (large photon number) the generalized $\mathrm{A}_{\text {- }}$ and $\mathrm{A}_{+}-$models lose the validity and, for a nonresolving photodetector and the considered classical states, the PSS (PAS) is better approximated by the generalized E-model $\left(\mathrm{E}_{+}\right.$-model), and the mean photon number necessarily decreases upon a photon subtraction. Thus, one may associate $E_{-}$used in [10] to the classical photodetection operator, in the same way as the bosonic annihilation operator $a$ is associated to the quantum photodetection operator.

In conclusion, the exact expressions of photon subtraction and addition superoperators, Eqs. (12) and (19), cannot always be written as $\gamma O \rho O^{\dagger}$ or $\gamma O^{\dagger} \rho O$, they reduce to these simple expressions only in some limits of the field intensity or for a photon-number resolving detector. More importantly, they depend on the form how detection is done, so the post-selected field state will depend essentially on the way the experimenter chooses to probe it.

\section{Acknowledgments}

SSM acknowledges financial support from $\mathrm{CNPq}$ and FAPESP, brazilian agencies. AVD would like to thank, in particular, the FAPESP, Grant No. 04/13705-3. 


\section{APPENDIX A: EXPRESSIONS FOR PHOTON SUBTRACTION}

(a) The coherent state $|\alpha\rangle$ is insensitive to the detector outcome, since it is an eigenstate of the lowering operator $a$ appearing in Eq. (10), so the resulting post-selected state is still a coherent state $|\alpha \cos \theta\rangle$, although having the field intensity attenuated. We get for the photon number distribution of the post-selected state

$$
p_{n, N \mathcal{D} k}^{\prime}=p_{n, R \mathcal{D} k}^{\prime}=e^{-n_{0} T}\left(n_{0} T\right)^{n} / n ! .
$$

The normalized factorial moments in the post-selected state are the same for both detectors,

$$
\langle n\rangle_{\mathcal{D} k} / n_{0}=T, \quad\langle n(n-1)\rangle_{\mathcal{D} k} / n_{0}^{2}=T^{2},
$$

although the subtraction probabilities associated to the detectors are different

$$
P_{R \mathcal{D} k}=e^{-n_{0} R} \frac{\left(n_{0} R\right)^{k}}{k !}, \quad P_{N \mathcal{D} k}=1-e^{-n_{0} R} \sum_{l=0}^{k-1} \frac{\left(n_{0} R\right)^{l}}{l !} .
$$

(b) For the thermal state the photon number distributions of the post-selected state for the $k$ photons resolving/nonresolving detector are

$$
\begin{aligned}
p_{n, R \mathcal{D} k}^{\prime}= & \left(\begin{array}{c}
n+k \\
n
\end{array}\right)\left(\frac{1+n_{0} R}{1+n_{0}}\right)^{k+1}\left(\frac{n_{0} T}{1+n_{0}}\right)^{n} \quad(\mathrm{~A} 4) \\
p_{n, N \mathcal{D} k}^{\prime}= & \left(\frac{1+n_{0} R}{n_{0} R}\right)^{k}\left[\frac{\left(n_{0} T\right)^{n}}{\left(1+n_{0} T\right)^{n+1}}\right. \\
& \left.-\sum_{l=0}^{k-1}\left(\begin{array}{c}
n+l \\
n
\end{array}\right)\left(\frac{n_{0} R}{1+n_{0}}\right)^{l} \frac{\left(n_{0} T\right)^{n}}{\left(1+n_{0}\right)^{n+1}}\right] .
\end{aligned}
$$

The $k$-photon subtraction probability is

$$
P_{\mathcal{D} k}=\frac{\left(n_{0} R\right)^{k}}{\left(1+n_{0} R\right)^{k+1-v}},
$$

and the normalized lowest factorial moments are

$$
\begin{gathered}
\frac{\langle n\rangle_{\mathcal{D} k}}{n_{0}}=T \frac{1+k+v n_{0} R}{1+n_{0} R} \\
\frac{\langle n(n-1)\rangle_{\mathcal{D} k}}{n_{0}^{2}}=T^{2} \frac{(1+k)(2+k)+2 v n_{0} R\left(2+k+n_{0} R\right)}{\left(1+n_{0} R\right)^{2}}
\end{gathered}
$$

where $v=1$ for the $N \mathcal{D} k$ and $v=0$ for the $R \mathcal{D} k$.

(c) For the 'mixed light' and the nonresolving detector $N \mathcal{D} k$, the resulting expressions are quite extensive, so we write out explicitly only the expressions for $k=1$,

$$
P_{N \mathcal{D} 1}=1-\frac{e^{-x}}{1+R n_{t}}
$$

$$
\begin{gathered}
\langle n\rangle_{N \mathcal{D} 1}=P_{N \mathcal{D} 1}^{-1} T\left[n_{0}-e^{-x} \frac{n_{0}+R n_{t}^{2}}{\left(1+R n_{t}\right)^{3}}\right] \\
\langle n(n-1)\rangle_{N \mathcal{D} 1}=P_{N \mathcal{D} 1}^{-1} T^{2}\left\{n_{0}^{2}+n_{t}\left(n_{0}+n_{c}\right)\right. \\
\left.-e^{-x} \frac{n_{0}^{2}+2 n_{c} n_{t}+n_{t}^{2}\left(1+4 R n_{0}+2 R^{2} n_{t}^{2}\right)}{\left(1+R n_{t}\right)^{5}}\right\},
\end{gathered}
$$

where $x \equiv R n_{c} /\left(1+R n_{t}\right)$. For the $R \mathcal{D} k$ the corresponding expressions are quite simple for any $k$

$$
\begin{aligned}
P_{R \mathcal{D} k} & =e^{-x} \frac{\left(R n_{t}\right)^{k}}{\left(1+R n_{t}\right)^{k+1}} L_{k} \\
\langle n\rangle_{R \mathcal{D} k} & =\frac{n_{t} T}{1+R n_{t}}\left[z-k \frac{L_{k-1}}{L_{k}}\right] \\
\langle n(n-1)\rangle_{R \mathcal{D} k} & =\left(\frac{n_{t} T}{1+R n_{t}}\right)^{2}\{z(1+z)-y(\mathrm{~A} \\
& \left.-\frac{2 k z L_{k-1}}{L_{k}}+\frac{k(k-1) L_{k-2}}{L_{k}}\right\}
\end{aligned}
$$

where $L_{k} \equiv L_{k}(y), y \equiv-n_{c} /\left[n_{t}\left(1+R n_{t}\right)\right]$ and $z \equiv$ $1+2 k-y$.

\section{APPENDIX B: EXPRESSIONS FOR SEQUENTIAL PHOTON SUBTRACTION}

For the sequential counting, in the simplest case, $k=2$, we obtain:

(a) For a coherent state $p_{n, \mathcal{S} 2}^{\prime}=e^{-n_{0} T^{2}}\left(n_{0} T^{2}\right)^{n} / n$ ! and

$$
\begin{gathered}
P_{\mathcal{S} 2}=e^{-n_{0} R(1+T)}\left(e^{n_{0} R T}-1\right)\left(e^{n_{0} R}-1\right) \\
\frac{\langle n\rangle_{\mathcal{S} 2}}{n_{0}}=T^{2}, \quad \frac{\langle n(n-1)\rangle_{\mathcal{S} 2}}{n_{0}^{2}}=T^{4} .
\end{gathered}
$$

(b) For a thermal state

$$
\begin{aligned}
p_{n, \mathcal{S} 2}^{\prime}= & g_{1}^{-1}\left[\frac{\left(n_{0} T^{2}\right)^{n}}{\left(1+n_{0} T^{2}\right)^{n+1}}-\frac{\left(n_{0} T^{2}\right)^{n}}{\left(1+n_{0} T\right)^{n+1}}\right. \\
& \left.-\frac{\left(n_{0} T^{2}\right)^{n}}{\left[1+n_{0}(1-R T)\right]^{n+1}}+\frac{\left(n_{0} T^{2}\right)^{n}}{\left(1+n_{0}\right)^{n+1}}\right]
\end{aligned}
$$

and

$$
P_{\mathcal{S} 2}=g_{1}, \quad \frac{\langle n\rangle_{\mathcal{S} 2}}{n_{0}}=T^{2} \frac{g_{2}}{g_{1}}, \quad \frac{\langle n(n-1)\rangle_{\mathcal{S} 2}}{n_{0}^{2}}=2 T^{4} \frac{g_{3}}{g_{1}},
$$

where

$g_{n} \equiv 1-\left[1+n_{0} R\right]^{-n}-\left[1+n_{0} R T\right]^{-n}+\left[1+n_{0} R(1+T)\right]^{-n}$.

These expressions are quite different from the corresponding expressions obtained in the appendix A. 


\section{APPENDIX C: EXPRESSIONS FOR PHOTON ADDITION}

(a) For a coherent state and the one-photon nonresolving detector we get

$$
\begin{gathered}
p_{n, N \mathbb{D} 1}^{\prime}=P_{N \mathbb{D} 1}^{-1} t e^{-n_{0}}\left[(r t)^{n} L_{n}\left(-\frac{n_{0}}{r}\right)-\frac{\left(n_{0} t\right)^{n}}{n !}\right](\mathrm{C} 1) \\
P_{N \mathbb{D} 1}=1-t e^{-r t n_{0}} \\
\frac{\langle n\rangle_{N \mathbb{D} 1}}{n_{0}}=\frac{1+r+r / n_{0}-t^{2} e^{-r t n_{0}}}{P_{N \mathbb{D} 1}} \\
\frac{\langle n(n-1)\rangle_{N \mathbb{D} 1}}{n_{0}^{2}}=\frac{t^{-2}+4 r /\left(n_{0} t\right)+2 r^{2} / n_{0}^{2}-t^{3} e^{-r t n_{0}}}{P_{N \mathbb{D} 1}},
\end{gathered}
$$

where $L_{n}(\cdot)$ is a Laguerre polynomial. For the $\mathrm{A}_{+}-$and $\mathrm{E}_{+-}$models the resulting expressions are

$$
\begin{aligned}
& \langle n\rangle_{\mathbb{A} 1} / n_{0}=1+n_{0}^{-1}+\left(n_{0}+1\right)^{-1} \\
& \langle n(n-1)\rangle_{\mathbb{A} 1} / n_{0}^{2}=1+4 n_{0}^{-1} \\
& \langle n(n-1)\rangle_{\mathbb{E} 1} / n_{0}^{2}=1+2 n_{0}^{-1} .
\end{aligned}
$$

(b) For a thermal state and the one-photon nonresolving detector one has

$$
p_{n, N \mathbb{D} 1}^{\prime}=P_{N \mathbb{D} 1}^{-1} t\left[\frac{\left(r t+n_{0}\right)^{n}}{\left(1+n_{0}\right)^{n+1}}-\frac{\left(n_{0} t\right)^{n}}{\left(1+n_{0}\right)^{n+1}}\right]
$$

$$
P_{N \mathbb{D} 1}=\frac{r t\left(1+n_{0}\right)}{1+n_{0} r t}
$$

$$
\begin{gathered}
\frac{\langle n\rangle_{N \mathbb{D} 1}}{n_{0}}=\frac{\left(t^{-1}+r / n_{0}\right)-\left[t /\left(n_{0} r t+1\right)\right]^{2}}{P_{N \mathbb{D} 1}} \\
\frac{\langle n(n-1)\rangle_{N \mathbb{D} 1}}{n_{0}^{2}}=2 \frac{\left(t^{-1}+r / n_{0}\right)^{2}-\left[t /\left(n_{0} r t+1\right)\right]^{3}}{P_{N \mathbb{D} 1}} .
\end{gathered}
$$

For the one-photon resolving detector we get

$$
\begin{aligned}
p_{n, R \mathbb{D} 1}^{\prime} & =\frac{\left(1+n_{0} r t\right)^{2}}{t n_{0}} n \frac{\left(n_{0} t\right)^{n}}{\left(1+n_{0}\right)^{n+1}}(\mathrm{C} 12) \\
P_{R \mathbb{D} 1} & =\frac{r t^{2}\left(1+n_{0}\right)}{\left(1+n_{0} r t\right)^{2}} \\
\frac{\langle n\rangle_{R \mathbb{D} 1}}{n_{0}} & =\frac{1+t+n_{0}^{-1}}{1+n_{0} r t} \\
\frac{\langle n(n-1)\rangle_{R \mathbb{D} 1}}{n_{0}^{2}} & =2 t \frac{2+t+2 n_{0}^{-1}}{\left(1+n_{0} r t\right)^{2}}
\end{aligned}
$$

The expressions for the $\mathrm{A}_{+}-$and $\mathrm{E}_{+^{-}}$models are

$$
\begin{aligned}
\langle n\rangle_{\mathbb{A} 1} / n_{0} & =2+n_{0}^{-1} \\
\langle n(n-1)\rangle_{\mathbb{A} 1} / n_{0}^{2} & =6+4 n_{0}^{-1} \\
\langle n(n-1)\rangle_{\mathbb{E} 1} / n_{0}^{2} & =2+2 n_{0}^{-1} .
\end{aligned}
$$

[1] R. J. Glauber, Phys. Rev. 130, 2529 ( 1963).

[2] M. D. Srinivas and E. B. Davies, Opt. Acta 28, 981 (1981).

[3] P. L. Kelley and W. H. Kleiner, Phys. Rev. 136, A316 (1964).

[4] B. R. Mollow, Phys. Rev. 168, 1896 (1968).

[5] N. Imoto, M. Ueda, and T. Ogawa, Phys. Rev. A 41, 4127 (1990).

[6] G. S. Agarwal, M. Graf, M. Orszag, M. O. Scully, and H. Walther, Phys. Rev. A 49, 4077 (1994).

[7] V. Peřinová and A. Lukš, Progress in Optics 40, ed. E. Wolf (Elsevier, Amsterdam, 2000), p. 115.

[8] A. V. Dodonov, S. S. Mizrahi, and V. V. Dodonov, Phys. Rev. A 72, 023816 (2005).

[9] Y. Ben-Aryeh and C. Brif, Preprint at quant-ph/9504009 (unpublished).

[10] M. C. de Oliveira, S. S. Mizrahi, and V. V. Dodonov, J. Opt. B: Quantum. Semiclass. Opt. 5, S271 (2003).

[11] A. V. Dodonov, S. S. Mizrahi, and V. V. Dodonov, J. Opt. B: Quantum. Semiclass. Opt. 7, 99 (2005).

[12] A. V. Dodonov, S. S. Mizrahi, and V. V. Dodonov, Phys. Rev. A 74, 033823 (2006).

[13] V. Parigi, A. Zavatta, M. Kim, and M. Bellini, Science
317, 1890 (2007).

[14] For $\mathcal{A}$ they were noticed already in the original paper [2]; see also [10, 11].

[15] M. Ban, Phys. Rev. A 47, 5093 (1993).

[16] J. Wei and E. Norman, J. Math. Phys. 4, 575 (1963); R. M. Wilcox, ibid. 8, 962 (1967).

[17] S. M. Chumakov, V. V. Dodonov, and V. I. Man'ko, J. Phys. A 19, 3229 (1986); S. V. Prants, ibid. 19, 3457 (1986); G. Dattoli, S. Solimeno, and A. Torre, Phys. Rev. A 34, 2646 (1986).

[18] R. R. Puri, Mathematical Methods of Quantum Optics (Springer, Berlin, 2001), p. 51.

[19] E. J. Gansen et al., Nature Photon. 1, 585 (2007); B. E. Kardynal, Z. L. Yuan, and A. J. Shields, ibid. 2, 425 (2008).

[20] M. J. Fitch, B. C. Jacobs, T. B. Pittman, and J. D. Franson, Phys. Rev. A 68, 043814 (2003); L. A. Jiang, E. A. Dauler, and J. T. Chang, ibid. 75, 062325 (2007).

[21] M. Martinelli and P. Martelli, Opt. Photonics News 19, No. 2, 31 (2008).

[22] G. Lacks, Phys. Rev. 138, B1012 (1965). 